\title{
Study of single muons with the Large Volume Detector at Gran Sasso Laboratory
}

\section{LVD Collaboration}

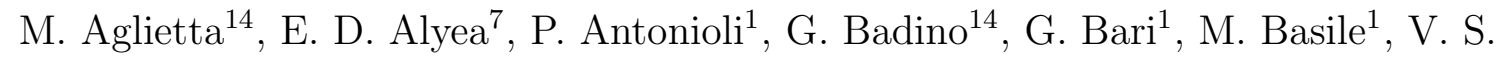
Berezinsky $^{9}$, F. Bersani ${ }^{1}$, M. Bertaina ${ }^{8}$, R. Bertoni ${ }^{14}$, G. Bruni ${ }^{1}$, G. Cara Romeo ${ }^{1}$, C. Castagnoli $^{14}$, A. Castellina ${ }^{14}$, A. Chiavassa ${ }^{14}$, J. A. Chinellato ${ }^{3}$, L. Cifarelli ${ }^{1 \dagger}$, F. Cindolo $^{1}$, A. Contin ${ }^{1}$, V. L. Dadykin ${ }^{9}$, L. G. Dos Santos ${ }^{3}$, R. I. Enikeev ${ }^{9}$, W. Fulgione $^{14}$, P. Galeotti ${ }^{14}$, P. Ghia ${ }^{14}$, P. Giusti ${ }^{1}$, F. Gomez ${ }^{14}$, R. Granella ${ }^{14}$, F. Grianti $^{1}$, V. I. Gurentsov ${ }^{9}$, G. Iacobucci ${ }^{1}$, N. Inoue ${ }^{12}$, E. Kemp ${ }^{3}$, F. F. Khalchukov ${ }^{9}$, E. V. Korolkova ${ }^{9}$ ๑, P. V. Korchaguin ${ }^{9}$, V. B. Korchaguin ${ }^{9}$, V. A. Kudryavtsev ${ }^{9 \dagger \dagger}$, M. Luvisetto $^{1}$, A. S. Malguin ${ }^{9}$, T. Massam ${ }^{1}$, N. Mengotti Silva ${ }^{3}$, C. Morello $^{14}$, R. Nania ${ }^{1}$, G.Navarra $^{14}$, L. Periale ${ }^{14}$, A. Pesci ${ }^{1}$, P.Picchi ${ }^{14}$, I. A. Pless ${ }^{8}$, V. G. Ryasny ${ }^{9}$, O. G. Ryazhskaya $^{9}$, O. Saavedra ${ }^{14}$, K. Saitoh ${ }^{13}$, G. Sartorelli ${ }^{1}$, M. Selvi $^{1}$, N. Taborgna ${ }^{5}$, V. P. Talochkin ${ }^{9}$, G. C. Trinchero ${ }^{14}$, S. Tsuji ${ }^{10}$, A. Turtelli ${ }^{3}$, P. Vallania ${ }^{14}$, S. Vernetto ${ }^{14}$, C. Vigorito ${ }^{14}$, L. Votano ${ }^{4}$, T. Wada ${ }^{10}$, R. Weinstein ${ }^{6}$, M. Widgoff ${ }^{2}$, V. F. Yakushev ${ }^{9}$, I. Yamamoto ${ }^{11}$, G. T. Zatsepin ${ }^{9}$, A. Zichichi ${ }^{1}$

1 University of Bologna and INFN-Bologna, Italy

2 Brown University, Providence, USA

3 University of Campinas, Campinas, Brazil

${ }^{4}$ INFN-LNF, Frascati, Italy

${ }^{5}$ INFN-LNGS, Assergi, Italy

6 University of Houston, Houston, USA

7 Indiana University, Bloomington, USA

8 Massachusetts Institute of Technology, Cambridge, USA

${ }^{9}$ Institute for Nuclear Research, Russian Academy of Sciences, Moscow, Russia

10 Okayama University, Okayama, Japan

11 Okayama University of Science, Okayama, Japan

12 Saitama University of Science, Saitama, Japan

13 Ashikaga Institute of Technology, Ashikaga, Japan

14 University of Torino and INFN-Torino, Italy

Institute of Cosmo-Geophysics, CNR, Torino, Italy

$\dagger$ now at the University of Salerno and INFN-Salerno, Italy

†† now at the University of Sheffield, UK

\footnotetext{
${ }^{a}$ Corresponding author, e-mail: korolkova@vaxmw.tower.ras.ru
} 


\begin{abstract}
The present study is based on the sample of $2.9 \times 10^{6}$ single muons observed by LVD at underground Gran Sasso Laboratory during 36500 live hours from June 1992 to February 1998. We have measured the muon intensity at slant depths from $3 \mathrm{~km}$ w.e. to $20 \mathrm{~km}$ w.e. Most events are high energy downward muons produced by meson decay in the atmosphere. The analysis of these muons has revealed the power index $\gamma$ of $\pi$ and $K$ spectrum: $\gamma=2.76 \pm 0.05$. The reminders are horizontal muons produced by the neutrino interactions in the rock surrounding LVD. The value of this flux near $90^{\circ}$ is $(6.1 \pm 2.7) \times 10^{-13} \mathrm{~cm}^{-2}$ $\mathrm{s}^{-1} \mathrm{sr}^{-1}$. The results are compared with Monte Carlo simulations and the world data.
\end{abstract}

Corresponding author: E.V. Korolkova, Institute for Nuclear Research of the Russian Academy of Science, 7a, 60th October Anniversary prospect, Moscow 117312, Russia Tel: 007095 9381930; $\quad$ Fax: 0070959385754.

E-mail: korolkova@vaxmw.tower.ras.ru 


\section{Introduction}

The study of atmospheric muons at large depth underground is the subject of experimental investigations due to the following reasons. First, muons and muonproduced secondary particles are the background for underground detectors designed to search for rare events including the tasks of neutrino and gamma-ray astronomy. Second, the calculations of atmospheric muon and neutrino fluxes are based on the hypothesis about primary cosmic-ray spectrum and hadron-hadron interactions. The existing deep underground detectors are not able to measure muon energy for direct deduction of energy spectrum. But they are able to measure muon 'depth-intensity' curve. This curve shows the vertical muons flux as a function of the rock (water, ice) depth and is related to the muon propagation through the rock, muon energy spectrum at sea level and then, to the primary cosmic-ray spectrum. The connection to the muon propagation allows tests of the cross-sections of muon interactions with the rock which have been used in the program for the muon transport.

The measurements of muon intensity using the single detector and technique from a relatively small depths to the large depth where neutrino-induced muons dominate are of special interest. Such an experiment observes muons at zenith angles from vertical to horizontal direction. The statistics for measurement of neutrino-induced flux in horizontal direction is small enough but the uncertainties in detecting the muon direction are absent. There is no need to suppress the flux of atmospheric muons by a factor of about $10^{6}$ using accurate time measurements.

The LVD structure and the complicated profile of the Gran Sasso mountains provide an opportunity to measure the muon 'depth-intensity' curve for slant depths from 3 to $20 \mathrm{~km}$ w.e. and the neutrino-induced muon flux at horizontal direction where the atmospheric muon flux is suppressed due to the large slant depth. The expected number of horizontal events caused by neutrino-induced muons is small (about one - two events per one LVD tower per year), therefore for the moment we cannot make a conclusion about neutrino oscillations.

In our previous paper [1] we have presented our first results on the measurement of the muon depth-intensity curve for the depth range of 3-20 km w.e. Since that time we have improved the criteria for event selection and increased the statistics. The analysis of Ref. [2 was based on the events with all multiplicities. Multiple muon events especially for large depths are more difficult to reconstruct than single muons. To avoid this problem we have also performed the analysis of single muons using stronger criteria of run and event selection. The analysis is based on an increased statistics compared with our previous publications.

In Section 2 the detector, the procedure of data analysis and conversion of the muon intensity to vertical are briefly described. In Section 3 the results of the analysis of the 'depth - vertical muon intensity' relation $\left(I_{\mu}(x)\right)$ are shown. In Section 4 we present the analysis of neutrino-induced events. Section 5 contains our conclusions.

\section{Detector and data selection}

The LVD (Large Volume Detector) has been described extensively elsewhere «1, 3, 田. The detector is located at Gran Sasso Laboratory, Italy. The minimal rock

overburden is $3 \mathrm{~km}$ w.e. LVD consists of 3 towers. Each tower is made of 38 modules with dimensions $2.1 \mathrm{~m} \times 6.2 \mathrm{~m} \times 1.0 \mathrm{~m}$. The data were obtained with the first LVD 
tower from June, 1992, when it was put into operation, until February, 1998. Total live time was 36500 hours. The tower has the size of $13 \mathrm{~m} \times 6.6 \mathrm{~m} \times 12 \mathrm{~m}$. Each module contains eight scintillator counters with active volume $1.0 \mathrm{~m} \times 1.5 \mathrm{~m} \times 1.0 \mathrm{~m}$ and mass of liquid scintillator of 1.2 tons, and tracking detector attached to the bottom and one vertical side of the supporting structure. Each tracking detector is made of 4 layers of tubes operating in limited streamer mode. Each layer has independent $x$ and $y$ readout strips. These established the $x$ and $y$ coordinates of the hits. The tracking system allows the measurements of particle direction with accuracy better than $0.5^{\circ}$.

The mountain structure above Gran Sasso laboratory allows the measurements of muons which traversed slant depth from 3 to more than $12 \mathrm{~km}$ w.e. The depths correspond to the median muon energies at sea level from 1.5 to $40 \mathrm{TeV}$ at zenith angles from $0^{\circ}$ to $90^{\circ}$. In the analysis we have used the sample of events containing only single muons. Events with all multiplicities are usually studied in the experiments with cosmic rays muons. Such an analysis supposes the accurate reconstruction of each event. The study of depth-intensity curve with all muon events observed by LVD has been presented in Ref. [2]. The multiple muons have been considered as independent muons and the acceptance for both single and multiple muons has been assumed the same. This is a good and well proven approximation for the derivation of the all-particle primary spectrum. The tasks requires however the reconstruction of muon events with all multiplicities and the measurements of the direction and slant depths with good accuracy. This is more difficult task for multiple muon events than for single ones. Most muons traverse small rock thicknesses. If the slant depth for a small fraction of these events is defined wrong, then the intensity in this direction will not change much. However these erroneously reconstructed events can contribute significantly to the muon intensity at large depth. To be sure in the precise event reconstruction in this analysis we have dealt with single muons only. The size of one LVD tower is small enough and more than 90\% of muon events are single muons. The number of muons in bundles is about $10-12 \%$ of the total number of muons. Possible uncertainties from neglecting multiple muons are less than or comparable to the errors from including multiple-muon events with erroneous reconstruction. In the case of single muon analysis we need to correct the absolute intensity for the number of unreconstructed events (multiple muons and muon induced cascades).

The trigger for muon events has been defined as follows: i) energy deposition is grater than $30 \mathrm{MeV}$ in at least two scintillator counters in two different modules; ii) hits in at least three layers in any three tracking detectors (hits in at least one layer per detector). The data runs have been selected as follows: runs have been accepted if they had more than one-hour duration and a counting rate is within a $15 \%$ range around the mean value for the set of runs. Moreover we have required at least 36 from 38 tracking modules and 240 from 304 scintillator counters of the first tower to be operated in any particular run. These criteria ensured the full and uniform acceptance of the detector. The final muon sample after these cuts consisted of 3151580 events. 2877659 (91\%) events have been reconstructed as single muons. Multiple muons and muons accompanied by cascades constituted $9 \%$. All reconstructed single muons were binned in two dimensional array with a cell size of $1^{\circ}$ in azimuthal angle $\phi$ and 0.01 in $\cos \theta$, where $\theta$ is the zenith angle. The accuracy of the reconstruction has been checked by observation of the Moon shadowing effect with single muon data [5]. It is better than $0.65^{\circ}$. 
The acceptance for each angular bin has been calculated using the simulation of muons traversing LVD taking into account the detector response. The thickness of rock crossed by the muon was determined from the mountain map.

The angular distribution $N_{\mu}(\phi, \cos \theta)$ obtained in the experiment has been converted to the 'depth-intensity relation' using the formula:

$$
I_{\mu}\left(x_{m}\right)=\frac{\sum_{i j} N_{\mu}\left(x_{m}\left(\phi_{j}, \cos \theta_{i}\right)\right)}{\sum_{i j}\left(S\left(x_{m}\left(\phi_{j}, \cos \theta_{i}\right)\right) \times \epsilon\left(x_{m}\left(\phi_{j}, \cos \theta_{i}\right)\right) \times \Omega_{i j} \times T\right)}
$$

where the summing up has been done over all angular bins $\left(\phi_{j}, \cos \theta_{i}\right)$ contributing to the depth $x_{m} ; S\left(x_{m}\left(\phi_{j}, \cos \theta_{i}\right)\right)$ is the cross-section of the detector in the plane perpendicular to the muon track at the angle $\left(\phi_{j}, \cos \theta_{i}\right) ; \epsilon\left(x_{m}\left(\phi_{j}, \cos \theta_{i}\right)\right)$ is the efficiency of muon detection and reconstruction; $\Omega_{i j}$ is the solid angle for the angular bin; $T$ is the live time. Angular bins with $\epsilon$ less than 0.03 were excluded from the analysis. The acceptance $A$ is defined as follows

$$
A(\cos \theta, \phi)=\epsilon(\cos \theta, \phi) \times \Omega(\cos \theta, \phi) \times S(\cos \theta, \phi)
$$

and is shown in Figure 1.

The intensity of muons at zenith angle $\theta$ was assumed to be related to the vertical intensity $I_{0}$ through the relation

$$
I\left(x_{m}, \theta\right)=I_{0}\left(x_{m}, \theta=0^{\circ}\right) / \cos \theta_{i}^{\star}
$$

where

$$
\cos \theta_{i}^{\star}=\frac{I_{\mu}^{c}\left(x_{m}, \cos \theta=1\right)}{I_{\mu}^{c}\left(x_{m}, \cos \theta_{i}\right)}
$$

is the ratio of calculated muon intensity at $\cos \theta=1$ to that at $\cos \theta_{i}$. This relation is valid for muons of atmospheric origin if we neglect the contribution of the prompt muons from charmed particles. According to the LVD data the ratio of prompt muons to pions does not exceed $2 \times 10^{-3}$ at $95 \%$ confidence level [6].

For depth - intensity relation the bin width of $200 \mathrm{~m}$. w. e. has been chosen. For depth more than $9 \mathrm{~km}$ w.e. we have chosen bins with the width of $500 \mathrm{~km}$ w.e. to increase the statistics for each bin. The conversion of muon intensity to the middle points of each depth bin has been done using formula:

$$
I_{\mu}^{m}\left(x_{i}\right)=I_{\mu}^{m}\left(x_{m}\right) \frac{I_{\mu}^{c}\left(x_{i}\right)}{I_{\mu}^{c}\left(x_{m}\right)}
$$

where $I_{\mu}^{m}\left(x_{m}\right)$ and $I_{\mu}^{c}\left(x_{m}\right)$ are the measured and calculated muon intensities at the weighted average depth $x_{m}$ which corresponds to the depth bin with the middle value of $x_{i} ; I_{\mu}^{m}\left(x_{i}\right)$ and $I_{\mu}^{c}\left(x_{i}\right)$ are the derived and calculated muon intensities at the depth $x_{i}$ which is the middle point of the depth bin. The values of $x_{m}$ have been obtained by averaging the depths for all angular bins contributing to the given depth bin with a weight equal to the detected number of muons. To calculate the muon intensities at $x_{m}$ and $x_{i}$ we have used the muon spectrum at sea level with previously estimated parameters [1, 2] (see also equation 7) and the simulated muon survival probabilities. Since the width of depth bins is quite small $(200 \mathrm{~m}$ w.e. for depth bins with high 
statistics) and the number of angular bins contributing to each depth bin is quite large (several hundreds), the conversion factor does not exceed $10 \%$.

\section{3. 'Depth - vertical intensity' relation in Gran Sasso rock}

To calculate the intensity of muons underground requires the intensity of muons at the surface as a function of energy and zenith angle, and the survival probability as a function of slant depth of rock traversed:

$$
I_{\mu}(x, \cos \theta)=\int_{0}^{\infty} P\left(E_{\mu 0}, x\right) \frac{d I_{\mu 0}\left(E_{\mu 0}, \cos \theta\right)}{d E_{\mu 0}} d E_{\mu 0}
$$

where $P\left(E_{\mu 0}, x\right)$ is the probability of muon with an initial energy $E_{\mu 0}$ at sea level to reach the depth $x$ and $d I_{\mu 0}\left(E_{\mu 0}, \cos \theta\right) / d E_{\mu 0}$ is the muon spectrum at sea level at zenith angle $\theta$. The intensity at the surface in the units of $\left(\mathrm{cm}^{2} \mathrm{~s} \mathrm{sr} \mathrm{GeV}\right)^{-1}$ can be approximated by [7]:

$$
\begin{aligned}
\frac{d I_{\mu 0}\left(E_{\mu 0}, \cos \theta\right)}{d E_{\mu 0}} & =A \times 0.14 \times E_{\mu 0}^{-\gamma} \\
& \times\left(\frac{1}{1+\frac{1.1 E_{\mu 0} \cos \theta^{\star}}{115 \mathrm{GeV}}}+\frac{0.054}{1+\frac{1.1 E_{\mu 0} \cos \theta^{\star}}{850 \mathrm{GeV}}}\right)
\end{aligned}
$$

where the values of $\cos \theta$ have been substituted by $\cos \theta^{\star}$ which have been taken from Ref. [8]. According to Ref. 8] $\cos \theta^{\star}=E_{\pi, K}^{c r}(\cos \theta=1) / E_{\pi, K}^{c r}(\cos \theta)$, where $E_{\pi, K}^{c r}$ are the critical energies of pions and kaons. Equation 7 has been obtained under a simple assumption of scaling in the high-energy hadron-nucleus interactions. Under this assumption the power index of primary spectrum, $\gamma$, is expected to be equal to that of meson (pion + kaon) spectrum, $\gamma_{\pi, K}$.

The muons were tracked trough the rock using propagation code MUSIC [9] to calculate the muon survival probabilities $P\left(E_{\mu 0}, x\right)$. The stochasticity of all processes of muon interaction with matter (nuclear interaction, pair production, bremsstrahlung and ionization) has been taken into account. The cross-sections were taken from Ref. [10, 11, 12]. The muon intensities calculated with bremsstrahlung cross-section from Ref. 12 are lower than those with bremsstrahlung cross-section from Ref. [13] which was used in our previous paper [1]. Using of the cross-section from Ref. [13] will result in a higher power index (softer muon spectrum) compared to the cross-section from Ref [12. The difference in power index is of order of 0.01 .

The measured 'depth - intensity' curve is shown in Figure 2 together with the best fit. The underground muon flux observed at a slant depth $x$ and zenith angle $\theta$ has the two-component nature and can be presented as:

$$
I_{\mu}(x, \theta)=I_{\mu}^{(\mu)}(x, \theta)+I_{\mu}^{(\nu)}(\theta)
$$

where $I_{\mu}^{(\mu)}(x, \theta)$ is the contribution of atmospheric muons and $I_{\mu}^{(\nu)}(\theta)$ denotes the contribution of muons from neutrino interactions in the rock surrounding detector. For slant depths of $13-20 \mathrm{~km}$ w.e. the muons seen in LVD are of the later origin. The last experimental point in Figure 2 corresponds to the neutrino-induced muon flux. This flux was measured at the depth of $13-20 \mathrm{~km}$ w.e. To convert the flux of neutrino-induced 
muons to vertical intensity we used the calculated ratio of horizontal and vertical fluxes of neutrino-induced muons which is equal to 2.1 at energy threshold of $1 \mathrm{GeV}$ for most models of atmospheric neutrino production:

$$
I_{\mu}^{\nu}\left(x, \theta=0^{\circ}\right)=I_{\mu}^{\nu}\left(x, \theta=90^{\circ}\right) / 2.1
$$

The 'depth - intensity' curve has been fitted with the calculated function with two free parameters: additional normalisation constant, $A$, and the power index of atmospheric pion and kaon spectrum, $\gamma$. As a result of the fitting procedure the following values of the free parameters have been obtained: $A=1.59 \pm 0.50, \gamma=2.76 \pm$ 0.05 for muon energies at sea level from 1.5 to $40 \mathrm{TeV}$. The errors of the parameters include both statistical and systematic uncertainties. The latter one takes into account possible uncertainties in the depth, rock composition, density and the uncertainty in the cross-sections used to simulate muon transport through the rock. These values are in good agreement with the results of similar analysis performed for muon events with all multiplicities observed by the first LVD tower during 21804 hours of live time: $A=1.95 \pm 0.50, \gamma=2.78 \pm 0.05$. Note that the estimates of the parameters $A$ and $\gamma$ are strongly correlated. The larger the value of $\gamma$ is, the larger the normalisation factor $A$ should be.

We have repeated the fitting procedure for restricted depth ranges. The results of this test are presented in Table 1. The results show that the power index is the same within errors for all depth ranges.

Neutrino-induced muon flux has not been included in fit procedure but has been added to the best fit at $2.5 \times 10^{-13} \mathrm{~cm}^{-2} \mathrm{~s}^{-1} \mathrm{sr}^{-1}$ level. Dashed curves in Figure 2 show possible values of muon intensities if we take into account uncertainties in the calculation of atmospheric neutrino spectrum at sea level, structure functions, corrections for quasielastic scattering and energy threshold of detector. The experimental value $(2.9 \pm 1.3) \times 10^{-13} \mathrm{~cm}^{-2} \mathrm{~s}^{-1} \mathrm{Sr}^{-1}$ is in agreement with the calculated one $(2.5 \pm 0.5) \times 10^{-13}$ $\mathrm{cm}^{-2} \mathrm{~s}^{-1} \mathrm{sr}^{-1}$. It also agrees with the compiled world results on underground muon intensities presented by Crouch in Ref. [14] where the flux of neutrino-induced muons is equal to $(2.17 \pm 0.21) \times 10^{-13} \mathrm{~cm}^{-2} \mathrm{~s}^{-1} \mathrm{sr}^{-1}$.

If the formula from Ref.[15] is used for the muon spectrum at sea level instead of eq. (可), the best fit value of $\gamma$ will be decreased by 0.04-0.05.

The value of $\gamma$ obtained with LVD data is in reasonable agreement with the results of other surface and underground experiments: DEIS [16], MUTRON [17], MIPhI [18] (the energies of these experiments correspond to first few points of our depth-intensity curve), ASD [19], NUSEX [20], MACRO [21], MSU [22] (if we consider the muon spectrum from 15] in the latter case). LVD data disagree with results of Baksan Scintillator Telescope and KGF [23, 24]. The difference here is likely due to the different methods of measurements, the applied analysis procedure in each experiment and uncertainties in the knowledge of overburden composition.

\section{Neutrino-induced muons.}

Let us describe the evaluation of horizontal neutrino-induced muon flux in more details. High energy neutrinos will produce high energy muons in the rock. These muons will have enough energy to traverse the entire detector. Reconstructed muons traversed rock thickness greater than $12 \mathrm{~km}$ w.e. have been considered as candidates 
for neutrino-induced muons. These depths correspond to the zenith angles more than $85^{\circ}$. We have recorded 95 such candidates during 36500 hours of LVD life time. A careful visual scan of all these tracks eliminated 5 candidates from the sample because of confusion in the pattern recognition.

Since the timing of the LVD experiment (12.5 ns) is not sufficient to determine the direction of a track crossing one tower there is a two-fold ambiguity in the direction for each measured track. In other words LVD cannot discriminate between muon direction $(\theta, \phi)$ and $\left(180^{\circ}-\theta, 180^{\circ}+\phi\right)$. For $\theta<90^{\circ}$ it is reasonable to assume that muons come from above, since the rock thickness above the horizon is smaller. Gran Sasso mountain has a very complicated profile and for many bins at $\theta \approx 90^{\circ}$ with $x>12 \mathrm{~km}$ w.e. the slant depth for inverse direction $x_{1}$ is appear to be less than $8 \mathrm{~km} \mathrm{w}$. e. The muon intensity for $8 \mathrm{~km}$ w.e. is 80 times greater than the intensity for $12 \mathrm{~km}$ w.e. In this case we assume that the muon came from the direction with smaller slant depth. Near horizontal muons with reconstructed slant depth greater $12 \mathrm{~km}$ w.e. and slant depth in opposite direction less than $8 \mathrm{~km}$ w.e. were excluded from neutrino-induced candidates and considered as bins $\left(\theta, 180^{\circ}+\phi\right)$. Totally we had 67 such events.

Some angular bins with slant depth greater than $12 \mathrm{~km}$ w.e. are surrounded by bins with smaller slant depths. According to the calculations of Ref. 99 the average angular deviation of muons is $0.45^{\circ}$ at $10 \mathrm{~km}$ w.e. and it is caused mainly by multiple Coulomb scattering. The probability of muon coming from the direction with smaller slant depth is greater. We have considered such a muon as coming from the direction with smaller depth assuming that it had been recorded into the bin with greater depth due to the reconstruction error or scattering.

Five muons produced in neutrino interactions with surrounding rock have survived all cuts for slant depths $x>13 \mathrm{~km} \mathrm{w}$. e., for $x>14 \mathrm{~km} \mathrm{w}$. e. we have found four such events and for $x>15 \mathrm{~km}$ w.e. there are two neutrino-induced muons.

A Monte Carlo has been used to estimate the expected number of neutrino-induced muons. The spectrum of neutrino-induced muons has been calculated following the formula:

$$
\frac{d N_{\mu}}{d E_{\mu}}=\int_{E_{\mu}}^{\infty} \frac{d N_{\nu}}{d E_{\nu}} \times \frac{P\left(E_{\nu}, E_{\mu}\right)}{d E_{\mu}} d E_{\nu}
$$

$d N_{\nu} / d E_{\nu}$ represents the neutrino spectrum, $P\left(E_{\nu}, E_{\mu}\right) / d E_{\mu}$ is the probability that a neutrino produces a muon in the interval $\left(E_{\mu}, E_{\mu}+d E_{\mu}\right)$. We have used the Bartol neutrino flux [25], which has a systematic uncertainty $\pm 14 \%$, and the Morfin and Tung [26], as well as Duke and Owens [27] parton distributions functions, which result in less than $1 \%$ difference in muon spectra. The major sources of uncertainties in the neutrino-induced muon flux are the uncertainty in the neutrino fluxes and neutrino cross-sections because of required extrapolations of the structure functions to small $x<<10^{-4}$. For neutrino-induced muons the calculations of atmospheric neutrino flux by various authors differ by as much as 17\%. Different standard parameterisation of charged current cross sections also differ by as much as $13 \%$ 28]).

Table 2 shows the number of muons observed by LVD during 36500 hours as well as calculated values (the uncertainty of calculations is $20 \%$ ).

The observed number of muons at large slant depth agrees with predictions within errors. The measured neutrino induced horizontal muon flux is $(6.1 \pm 2.7) \times 10^{-13}$ $\mathrm{cm}^{-2} \mathrm{~s}^{-1} \mathrm{sr}^{-1}$, while the calculated one is $(5.2 \pm 1.1) \times 10^{-13} \mathrm{~cm}^{-2} \mathrm{~s}^{-1} \mathrm{sr}^{-1}$. Our 
measured value agrees with the results of other experiments: Soudan-2 (5.00 $\pm 0.55 \pm$ $0.51) \times 10^{-13} \mathrm{~cm}^{-2} \mathrm{~s}^{-1} \mathrm{sr}^{-1}$ [29], Frejus (the flux recalculated for our energy threshold) $(4.77 \pm 0.86) \times 10^{-13} \mathrm{~cm}^{-2} \mathrm{~s}^{-1} \mathrm{sr}^{-1}$ [30], in South Africa mine $(4.59 \pm 0.42) \times 10^{-13}$ $\mathrm{cm}^{-2} \mathrm{~s}^{-1} \mathrm{sr}^{-1} 31$.

\section{Conclusions}

We have measured the underground muon intensity as a function of the slant depth in the range of $3-20 \mathrm{~km}$ w.e. The analysis of 'depth-intensity' relation in the depth range $3-12 \mathrm{~km}$ w.e. has been done to obtain the power index of differential energy spectrum of the pions and kaons in the atmosphere $\gamma=2.76 \pm 0.05$ in the energy range of 1 - $40 \mathrm{TeV}$. The errors include both statistical and systematic uncertainties with the systematic error due to the uncertainty of the muon interaction cross-sections dominating. Our results are in good agreement with other experiments. Muons traversed slant depth more than $13 \mathrm{~km}$ w.e. were analysed to obtain the horizontal flux of neutrino-induced muons. This flux is equal to $(6.1 \pm 2.7) \times 10^{-13} \mathrm{~cm}^{-2} \mathrm{~s}^{-1} \mathrm{sr}^{-1}$ and is consistent with our calculations and reported results of other experiments. Our fit to this data at the 'depth-intensity' curve is in a good agreement with the fit of Crouch, which is a summary of various experiments.

\section{Acknowledgments}

The Collaboration wishes to thank the staff of Gran Sasso Laboratory for assistance. This work is supported by the Russian Ministry of Industry, Science and Technologies, the Italian Institute for Nuclear Physics and the Russian Found for Basic Research (grant 00-02-16112).

\section{References}

[1] LVD Collabotarion (M. Aglietta et al.), Astroparticle Phys. 3 (1995) 311.

[2] LVD Collaboration (M. Aglietta et al.), Phys. Rev. D 58 (1998) 092005.

[3] LVD Collaboration (G. Bari et al.), Nuclear Instruments and Methods in Physics Research. A264 (1988) 5.

[4] LVD Collaboration (G. Bari et al.), Nuclear Instruments and Methods in Physics Research. A277 (1989) 11.

[5] LVD Collaboration (M. Aglietta et al.), presented by E.Korolkova, Proc. 26 Intern. Cosmic Ray Conf. (Salt Lake city) 7 (1999) 218.

[6] LVD Collaboration (M. Aglietta et al.), Phys. Rev. D 60 (1999) 112001.

[7] T. K. Gaisser, Cosmic Rays and Particle Physics (Cambridge University Press, 1990)

[8] L. V. Volkova, Preprint Lebedev Physical Institute N 72 (1969). 
[9] P. Antonioli et al., Astroparticle Phys. 7 (1997) 357.

[10] L. B. Bezrukov and E. V. Bugaev, Proc. 17th Intern. Cosmic Ray Conf. (Paris) 7 (1981) 90.

[11] R. P. Kokoulin and A. A. Petrukhin, Proc. 12th Intern. Cosmic Ray Conf. (Hobart) 6 (1971) 2436.

[12] S. R. Kelner, R. P. Kokoulin, and A. A. Petrukhin, Physics of Atomic Nuclei 60 (1997) 576.

[13] L. B. Bezrukov and E. V. Bugaev, Proc. 17th Intern. Cosmic Ray Conf. (Paris) 7 (1981) 102.

[14] M. Crouch, Proc. 20th ICRC (Moscow) 6 (1987) 165.

[15] L. V. Volkova, G. T. Zatsepin, and L. A. Kuzmichev, Sov. J. Nucl. Phys. 29 (1979) 1252 .

[16] O. C. Allkofer et al., Proc. 17th Intern. Cosmic Ray Conf. (Paris) 10 (1981) 321.

[17] S. Matsuno et al., Phys. Rev. D 29 (1984) 1.

[18] V. D. Ashitkov et al., Proc. 19th Intern. Cosmic Ray Conf. (La Jolla) 8 (1985) 77.

[19] F. F. Khalchukov et al., Proc. 19th Intern. Cosmic Ray Conf. (La Jolla) 8 (1985) 12; R. I .Enikeev et al., Sov. J. Nucl. Phys. 47 (1988) 1044.

[20] G. Battistoni et al., Nuovo Cimento 9C (1986) 196.

[21] M. Ambrosio et al. (MACRO Collaboration), Phys. Rev. D 52 (1995) 3793.

[22] N. P. Il'ina et al., Proc. 24st Intern. Cosmic Ray Conf. (Rome) 1 (1995) 524.

[23] Yu. M. Andreyev, V. I. Gurentsov, and I. M. Kogai, Proc. 20th Intern. Cosmic Ray Conf. (Moscow) 6 (1987) 200.

[24] M. R. Krishnaswami et al., Proc. 18th Intern. Cosmic Ray Conf. (Bangalore) 11 (1983) 450.

[25] V. Agraval, T. K. Gaisser, P. Lipari, T. Stanev, Phys. Rev. D 53 (1996) 1314.

[26] J. G. Morfin, W. K. Tang Zeitchift fur Physics C 52 (1991) 13.

[27] D. W. Duke, J. F. Owens, Phys. Rev. D 30 (1984) 49.

[28] T. Gaisser, F. Halzen, T. Stanev, Phys. Reports 258 (1995) 173.

[29] D. Demuth and M. Goodman, Proc. 27th ICRC (Hamburg) HE (2001) 1090.

[30] W. Rhode et al., Astroparticle physics 4 (1996) 217.

[31] M. Crouch et al., Phys. Rev. 18 (1978) 2239. 
Table 1: The value of power index of meson spectrum for various depth ranges. The errors are statistical only.

\begin{tabular}{|c|c|c|}
\hline Depth interval, km w.e. & $\gamma$ & $\chi^{2} /$ d.o.f. \\
\hline $3-12$ & $2.76 \pm 0.02$ & $25.8 / 34$ \\
$4-12$ & $2.78 \pm 0.03$ & $19.0 / 29$ \\
$5-12$ & $2.79 \pm 0.04$ & $16.8 / 24$ \\
$6-12$ & $2.82 \pm 0.06$ & $14.8 / 19$ \\
$7-12$ & $2.94 \pm 0.14$ & $11.4 / 14$ \\
$8-12$ & $2.76 \pm 0.22$ & $6.7 / 9$ \\
$9-12$ & $2.60 \pm 0.50$ & $3.3 / 4$ \\
\hline
\end{tabular}

Table 2: The number of muons at large depths observed by LVD $\left(N_{\text {obs }}\right)$ and calculated values $\left(N_{\mu}^{a t m}\right.$-atmospheric muons, $N_{\mu}^{\nu}$ - neutrino induced muons, $\left.N_{\mu}^{t o t}=N_{\mu}^{a t m}+N_{\mu}^{a t m}\right)$.

\begin{tabular}{|c|c|c|c|c|}
\hline$h$, km.w.e. & $N_{\mu}^{\text {atm }}$ & $N_{\mu}^{\nu}$ & $N_{\mu}^{\text {tot }}$ & $N_{\text {obs }}$ \\
\hline$h>13$ km.w.e. & 0.45 & 3.75 & 4.20 & 5 \\
$h>14$ km.w.e. & 0.31 & 2.92 & 3.23 & 4 \\
$h>15$ km.w.e. & 0.03 & 1.50 & 1.53 & 2 \\
\hline
\end{tabular}




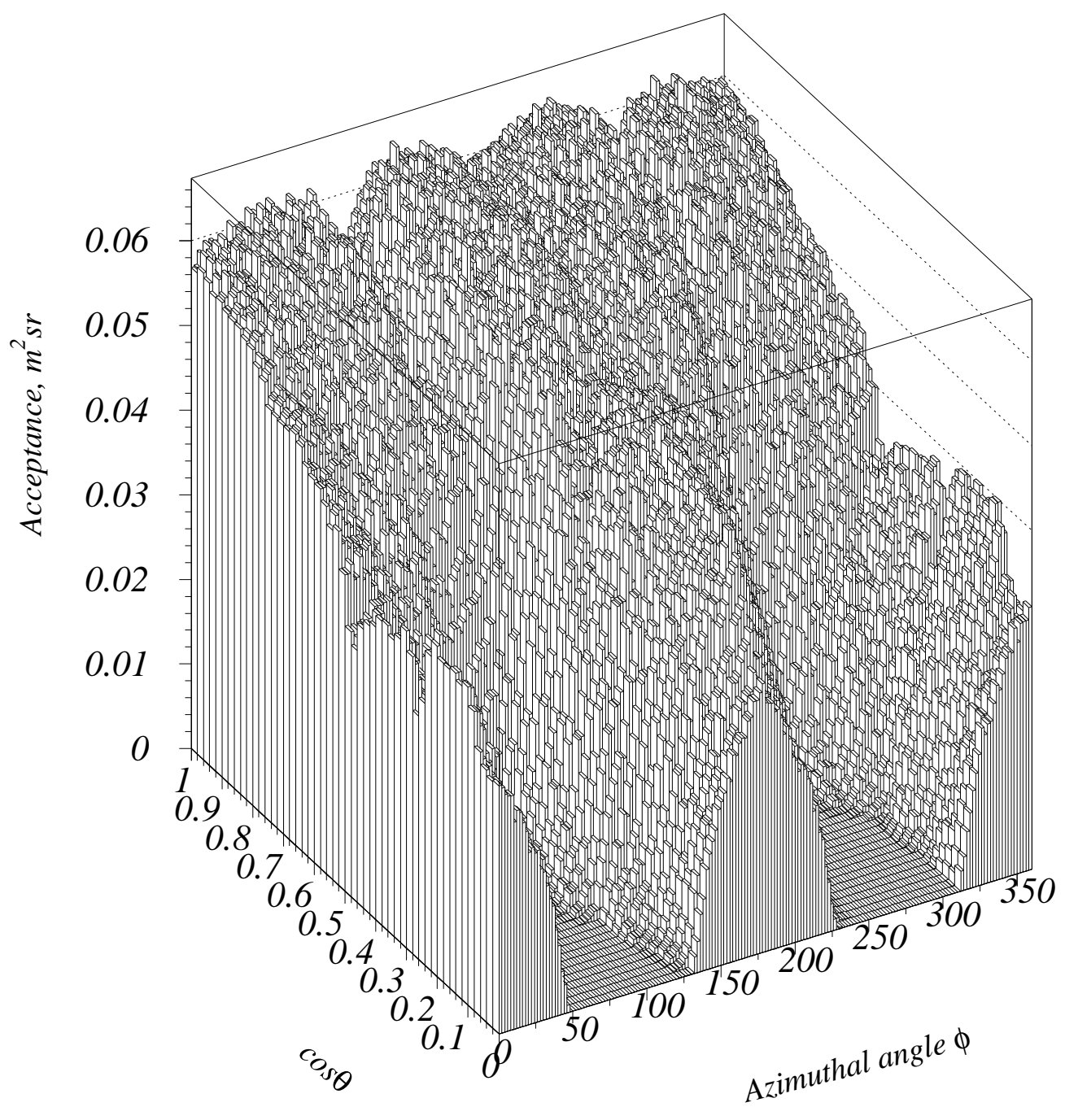

Figure 1: The LVD acceptance for single muons as a function of the cosine of the zenith angle $\theta$ and azimuthal angle $\phi$. The angular cell for this plot was chosen as 0.02 $(\cos \theta) \times 2^{\circ}(\phi)$. 


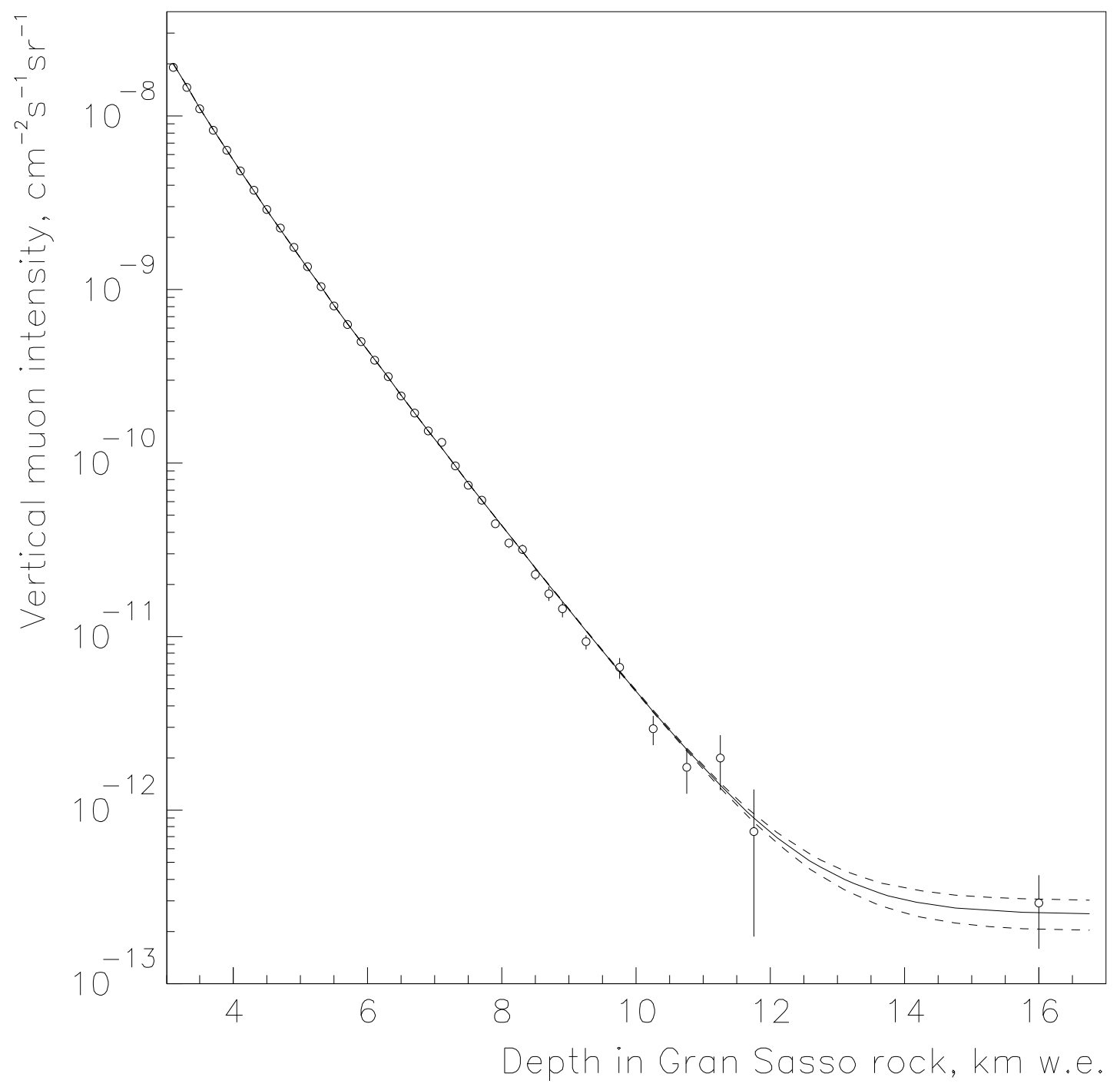

Figure 2: 'Depth - vertical muon intensity' relation in Gran Sasso rock. LVD data are presented together with the best fit (solid curve). Dashed curves show the calculated intensities for maximal and minimal contributions from neutrino-induced muons (see text for details). 